be based on the hypothesis that doses near to the toxic level will carry dramatic benefit that cannot be obtained with a lower loading dose-or more gradually on conventional maintenance doses of digoxin, which achieve the same effect in a few days. ${ }^{13}$ Control of emergency situations such as pulmonary oedema can usually be achieved by diuretic therapy and artificial ventilation if necessary; the added benefit of digitalis treatment is unlikely to be dramatic; attempts to obtain a maximal effect of digitalis treatment are likely to lead to toxicity and should be avoided. To give a "full digitalising dose" to a patient with heart failure in sinus rhythm without detailed consideration of all the problems is a recipe for disaster. The therapeutic margin is very narrow: indeed it has been argued that if digoxin were to be introduced as a new drug today it would be regarded as too toxic for clinical use.

\footnotetext{
1 Smith, T. W., Butler, V. P., and Haber, E., New England fournal of Medicine, 1969, 281, 1212.

2 Smith, T. W., and Haber, E., fournal of Clinical Investigation, 1970, 49,

3 Chamberlain, D. A., et al., British Medical fournal, 1970, 3, 429.

4 Coltart, J., Howard, M., and Chamberlain, D., British Medical fournal, $1972,2,318$.

5 Peck, C. C., et al., New England Fournal of Medicine, 1973, 289, 441.

6 Carruthers, S. G., Kelly, J. G., and McDevitt, D. G., British Heart fournal, 1974, 36, 707.

Whiting, B., Summer, D. J., and Goldberg, A., Scottish Medical fournal,

1973, 18, 69.
${ }^{2}$ Weintraub, M., Au, W. Y. U., and Lasagna, L., fournal of the American Medical Association, 1973, 224, 481.

9 Shaw, T. R. D., Howard, M. R., and Hamer, J., Lancet, 1972, 2, 303.

10 Daw, T. R. D., Howard, M. R., and Hamer, J., Lancet, 1972, 2, 303.

11 Gouley, B. A., and Scloff, L., American Heart fournal, 1938, 16, 561.

12 Knoebel, S. B., in Digitalis, ed. C. Fisch and B. Surawicz, p. 121. 1969.

13 Marcus, F. J., et al., Circulation, 1968, 34, 865.
}

\section{Black Eyes and Blow-out Fractures}

Black eyes are common enough after fights, sports injuries, and accidents, but there is no really effective treatmentthe time-honoured beefsteak is both expensive and useless and most of the drugs said to reduce haematomas are not of convincing value.

The important clinical problem is whether a blow-out fracture of the orbit is present. The eye is protected by the bones of the orbit. Blunt trauma, such as from a fist or a ball, tends to force the eye back into the orbit and the hydraulic force may cause the bones to give way in the thinnest place, which is usually in the floor of the orbit. Generally the bones of the orbital margin are intact. Sometimes when the floor is fractured orbital fat and the inferior ocular muscles may herniate through and become incarcerated; the patient will then experience double vision, unless his eye is occluded by the bruised and swollen lid. The double vision will be most marked on attempted vertical gaze. Diplopia may occur as a result of an orbital haematoma without any associated fracture, but this symptom should always lead one to suspect bone damage. The other features of a blow-out fracture include vertical displacement of the eye (easy to measure by using a straight edge and comparing the levels of the corneal margin between the two eyes) and some degree of enophthalmos owing to the reduction of the orbital contents. However, this may be difficult to gauge if the bruising is extensive. The presence of orbital emphysema is not diagnostic of a blow-out fracture but merely suggests that the neighbouring sinuses have been injured. The infraorbital nerve, which supplies the sensory innervation for the lower lid and upper part of the cheek, travels through a bony tunnel in the floor of the orbit. In a blow-out fracture this nerve is often injured, producing numbness in this region. There are, then, no clinical features which guarantee the diagnosis of a blow-out fracture, but the general clinical picture and in particular the presence of diplopia should alert one to the possibility.

Standard skull $x$-rays frequently fail to show the fracture, so if the condition is suspected it is wise to seek advice from the radiologist. Both anterior-posterior and occupito-mental views may be required, as well as the use of tomography. Suggestive signs include opacification of either the maxillary or ethmoid sinuses on the affected side. The herniation of the orbital contents may sometimes be seen in what is known as the "hanging drop" pattern, and in other cases discontinuity of the orbital floor can be clearly shown.

If a blow-out fracture is part of a serious injury to the skull, then its treatment should take a secondary place in the management of the patient's general condition. If the patient is fit, however, treatment should be undertaken urgently. It is not the fracture itself which is important but the fact that the inferior ocular muscles are affected, for if these are not freed within a few days of the injury permanent diplopia may result. The orbital floor represents a demarcation zone between ophthalmologists and E.N.T. surgeons, both of whom may claim expertise in this area-but in fact the treatment is probably best conducted by faciomaxillary surgeons used to handling large numbers of traumatic cases. ${ }^{1}$ Before surgery is undertaken it is wise to perform a forced duction test under anaesthesia to be sure that the muscles are trapped. The muscles can be freed either by an approach along the orbital floor or from below through the maxillary sinus. In some instances all that is required is the freeing of the muscles and elevation of the bone, while in others the defect may need to be covered with a piece of silicone sheeting.

${ }^{1}$ Murphy, A. G., Fournal of the Royal Army Medical Corps, 1974, 120, 40.

\section{Alcohol and the Small Bowel}

Chronic alcoholics are prone to suffer from a variety of gastrointestinal disorders, many of which are well known and have been widely studied-gastritis, the Mallory-Weiss syndrome, pancreatitis, various forms of liver disease, and mucous colitis. They also suffer an increased incidence of gallstones and duodenal ulceration, though opinions differ on the effect of alcohol on acid secretion by the stomach. Symptoms such as anorexia, vomiting, and abdominal discomfort are common, and the frequency of diarrhoea, which may sometimes amount to frank steatorrhoea, suggests that alcohol may interfere with small-bowel function. Various degrees of malabsorption of fat, nitrogen, vitamin $B_{12}$, and folate can in fact be shown in some alcoholics without obvious liver or pancreatic disease, and defective $D$-xylose excretion is frequent. ${ }^{1-4}$ Though one or more indices of absorption can be found to be affected in most patients $-83 \%$ in one series 2 -they do not necessarily parallel each other, and it is likely that several mechanisms are responsible.

One possibility, in view of the abnormal D-xylose tests, is that alcohol might damage the jejunum; but jejunal biopsies have generally been reported to be normal ${ }^{3} 4$ though megaloblastosis has been noted in the epithelial cells of crypts and 
villi in folate-deficient alcoholics, ${ }^{56}$ and ultrastructural changes similar to those found in the liver have been seen on electron microscopy. ${ }^{7} \mathrm{~A}$ direct toxic effect on the small bowel, however, is unlikely to explain ileal dysfunction (as shown by defective absorption of vitamin $\mathrm{B}_{12}$ ), since alcohol is rapidly and completely absorbed in the upper gut. Both oral and intravenous alcohol cause a sharp fall in mixing (type I) contractions in the jejunum and an increase in propulsive (type III) waves in the ileum when given to either chronic alcoholics or healthy volunteers. ${ }^{8}$ An indirect stimulant action either of alcohol or its metabolites is therefore possible.

An increasingly recognized accompaniment of alcoholism is folate deficiency, and this may be a cause of malabsorption in some patients, since the defect can be cured by providing folate supplements even though intake of alcohol is continued. ${ }^{4}$ Protein deficiency may be another. A pattern of excessive loss of fat and nitrogen with normal levels of $D$-xylose and vitamin $B_{12}$ may be found, suggesting pancreatic insufficiency, and half of a group of patients studied by Mezey et al. ${ }^{3}$ had defective pancreatic function as judged by the results of a secretin test. In some this could be reversed by increasing protein consumption in spite of continued intake of alcohol, while in others the lack of response was attributed to irreversible damage to the pancreas (though there were no symptoms of pancreatitis). Alcohol ingestion does not apparently directly affect pancreatic function, and the malabsorption may be due to dietary protein lack; similar effects on pancreatic function have been seen in kwashiorkor and adult malnutrition.

Diarrhoea in the alcoholic may be due to structural changes in the small or large bowel, in the pancreas, or in the liver. It may result from interference in gastrointestinal function, altering motility or causing an imbalance of fluid, electrolyte, and nutrient absorption. It may be secondary to dietary deficiencies of protein, folate, and possibly other essential substances. In the individual patient it is necessary to try and unravel these different mechanisms in order to find the right treatment.

\footnotetext{
1 Small, M., Longarini, A., and Zamcheck, N., American fournal of Medicine, $1959,27,575$.

Roggin, G. M., et al., fohns Hopkins Medical fournal, 1969, 125, 321.

3 Mezey, E., et al., Gastroenterology, 1970, 59, 657.

Halsted, C. H., Robles, E. A., and Mezey, E., Gastroenterology, 1973, 64, 526.

5 Bianchi, A., et al., New England fournal of Medicine, 1970, 282, 859.

- Hermos, J. A., et al., Annals of Internal Medicine, 1972, 76, 957.

7 Rubin, E., et al., Gastroenterology, 1972, 63, 801.

8 Robles, E. A., et al., fohns Hopkins Medical fournal, 1974, 135, 17.
}

\section{Genitourinary Medicine}

During the past 25 years there has been a great expansion of the venereal diseases service in Britain. Not only has the number of new cases increased more than threefold, so that in 1973 more than 360000 cases were seen at the clinics, but the type of disease diagnosed has also changed radically. Syphilis and gonorrhoea, the two conditions for which the clinics were originally instituted 58 years ago, now account for less than $25 \%$ of the cases diagnosed, and most of the work is concerned with other sexually transmitted diseases and conditions which arise as a result of sexual activity or affect the genitourinary tract. In the more progressive centres the breadth of the specialty has increased steadily and it now includes a wide spectrum of medical problems.
In Britain and several other western countries patients who develop symptoms after sexual activity usually seek out a clinic for sexually transmitted diseases and do not go to their general practitioners. The clinics are staffed by doctors, nurses, social workers, technicians, and other workers who have received special training in the subject. They have at their disposal specialized diagnostic equipment as well as the knowledge and experience of how to interpret the results. Contact tracers, working in the clinics in close collaboration with the doctors, play a major role in the control of the incidence of the diseases by helping the patients with their contacts and also reminding defaulters who have not completed their treatment or follow-up. Arrangements are usually available for patients with personal, family, social, or financial problems to receive help from a medical social worker, and in a few centres a psychiatrist attends the clinic for one or two sessions a week. The grouping of infectious diseases of the genital and urinary tracts together in one specialty also facilitates teaching of the subject to undergraduate and postgraduate students and is a stimulus for research.

Over the years the facilities for treatment of sexually transmitted disease in Britain have become the most highly developed in the world, and foreign postgraduate students and workers frequently visit the larger centres. Furthermore, the British Postgraduate Medical Federation now organizes a successful postgraduate course in sexually transmitted diseases twice a year.

A recent publication ${ }^{1}$ by the Office of Health Economics points out that the United Kingdom has a lower rate of syphilis and gonorrhoea per 100000 population than any other comparable industrial country and suggests that, at approximately $£ 5$ million per annum, the cost-effectiveness of the service is satisfactory. However, the report points out that there would be many advantages if the specialty of venereology could be broadened to include a wider range of genitourinary disorders and the name changed to genitourinary medicine.

Those who would benefit most from such a change are the patients themselves, as most of them do not have a venereal disease in the old legal sense of the term. It would, therefore, help to remove some of the social stigma of attending a clinic, make it easier for general practitioners to refer patients with genitourinary symptoms, and encourage more young doctors to make a career in the specialty. It has been apparent to a minority of those working in the field for several years that as the subject has evolved the older names for the specialty were too narrow and terms such as V.D. clinic or special clinic had become increasingly inappropriate. The term sexually transmitted diseases may be satisfactory to describe the diseases themselves but is unsatisfactory for the name of the department, the specialty itself, and the doctors who work in it.

Genitourinary medicine accurately describes the work now carried out in the clinics and has no emotional or judgemental overtones. There should be no confusion with urology, which is a surgical specialty concerned with the same anatomical areas, just as cardiology is not confused with cardiac surgery or neurology with neurosurgery. The new name simply acknowledges a situation which already exists and is part of the redefinition of medical specialties which has been occurring during the past decade in such subjects as rheumatology and communicable diseases. As a result of the change the work of genitourinary physicians could expand to include infertility and other diseases of the genitourinary tract when appropriate. New departments would be situated in the general outpatient concourse and not be physically isolated as many are still today. In this regard it is interesting to note that at the St. Louis Hospital in Paris the name of the department had to be changed 